\title{
A new ultrasound and clinical classification for management of prostatic abscess
}

\author{
Lucio Dell'Atti \\ Department of Urology, University Hospital "St. Anna”, Ferrara, Italy
}

\begin{abstract}
Summary Objectives: In literature, most of the published data regarding prostatic abscess (PA) are case reports, whereas there is no standardization of the diagnostic and therapeutic routines. The purpose of this study is a new classification of ultrasound imaging of PA with clinical features correlation.

Material and Methods: We retrospectively analysed the ultrasound database archives and performed a MEDLINE® research of the peer reviewed literature on diagnosis and case reports of PA using the terms "prostate and abscess". Results: PA can be classified into five Types: Type I - PA is present focally in a prostate lobe $(\leq 10 \mathrm{~mm})$. Type II - PA is present in a prostate lobe $(>10 \mathrm{~mm})$ and/or partially overcrosses the border of the midline prostatic glandular.

Type III - PA is present in both glandular lobes form of multifocal areas $(\leq 10 \mathrm{~mm})$. Type IV - PA is present in both glandular lobes form of multifocal areas ( $>10 \mathrm{~mm}$ ).

Type V - PA involving intra or extraprostatic structures (bladder, urethra, seminal vesicles and prostatic capsule). The different ultrasound imaging and diagnostic criteria are listed for each type and subtype.

Conclusions: The sonographic pattern of PA is usually characteristic and easily differentiated from other glandular lesions. The purpose of the study was to associate the use of TRUS to a clinical standardized classification in order to facilitate PA diagnosis and localization directing the clinician treatment to the correct management and adequate therapeutic treatment.
\end{abstract}

KEY WORDS: Prostatic Abscess; Transrectal Ultrasound; Classification.

Submitted 27 February 2015; Accepted 30 April 2015

\section{INTRODUCTION}

Prostatic abscess (PA) in an unusual condition with an incidence of about $0.5 \%$ of all prostatic disorders (1). This prostatic disease is thought to arise most commonly as a consequence of inadequately treated acute bacterial prostatitis. Other causes include bladder outlet obstruction such as: benign prostatic hypertrophy, indwelling urethral catheters and lower urinary tract invasive procedures as prostate biopsy $(2,3)$.

Immunosuppressed states and diabetes mellitus predispose to the formation of PA (4). The signs and symptoms of PA are non-specific and include urinary retention, dysuria, a palpably enlarged and tender prostate gland, and fever. Several studies employed in PA diagnosis include transrectal ultrasound (TRUS), computed tomography (CT) scanning, or magnetic resonance imaging (MRI) (5). However, TRUS is the most widely employed imaging modality for PA and the best technique executable in a short time, with low radiant exposure, and low costs at detecting smaller abscess (6). To date, in the literature there is no specific classification based on imaging, and clinical aspects of different types of prostatic abscess. In this study we present a review of PA ultrasound imaging and propose a new classification of PA related to clinical features of the patients.

\section{MATERIALS AND METHODS}

We retrospectively analysed the ultrasound database at our Department of Urology, which contains the stored images of 4000 cases from August 2007 to December 2014. Only 1300 of these images (33\%) are TRUS to study prostate diseases. All cases were evaluated by an expert urologist (LD). Each patient was treated under local anesthesia with lidocaine spray $(10 \mathrm{gr} / 100 \mathrm{ml})$, applied two minutes before the TRUS (7). The procedure was performed with the patient in the left lateral decubitus using a General Electric Logiq 7 equipped with a 5-9 $\mathrm{MHz}$ multi-frequency convex probe "end-fire". Each TRUS examination included an assessment of the prostatic diameter, the measurement of the whole prostate volume, and the evaluation of the transition zone, capsular and seminal vesicle characteristics, as well as the morphological description of potential pathological features. On 1 January, 2015 a MEDLINE ${ }^{\circledR}$ search of the peer reviewed literature on diagnosis and case reports of PA was done using the terms "prostate and abscess". A total of 47 papers were evaluated. We propose a classification of prostatic abscess into 5 distinct types according to the literature and validated by our experience.

\section{RESULTS}

Type I - Prostatic abscess is present focally in a prostate lobe $(\leq 10 \mathrm{~mm})$.

If the prostatic abscess interests focally $(\leq 10 \mathrm{~mm})$ one prostate lobe (Type I), frequently is present also in the transition zone and in the central zone of the prostate $(2,4)$. We can divide it into three subtypes (IA, IB and IC) on the basis of ultrasonography (US) pattern and the type of clinical symptoms. In the subtype IA, PA presents a discrete low echoic or anechoic area with irregular contour. This contour shows along the outermost periphery 
a diffuse enlargement with a homogeneous low level of echogenicity due to oedema and inflammatory cell infiltration. This subtype of PA is incidentally diagnosed during a routine ultrasound for diagnostic purposes or follow-up for different urologic problems (urinary retention, urinary tract infection, chronic indwelling catheter, prostate biopsy). These subtypes must be differentiated from the prostatic retention cyst, which is frequently $(0.5-7.9 \%)$ observed in the gland as an isolated lesion surrounded by normal tissue. Ultrasound features are an anechoic content (less than $8 \mathrm{~mm}$ diameter) with thin and smooth walls or threadlike septa (8).

In the subtype IB, PA presents a non-homogeneous content with well-defined edges and/or irregular contour (with possible thickened aspect for homogeneous low level echogenicity or hyperechoic structure). This nonhomogeneous content like fluid material within prostatic abscess shows a movement by changing the position of the patient. In the antibiotic era, Gram-negative bacilli (mainly E. coli) cause about 60 to $80 \%$ of cases. Other significant pathogens include Pseudomonas species, Staphylococcus species, and occasionally obligate anaerobic bacteria (9). In the subtype IC, PA can appear with the characteristics of both types IA and IB, but is diagnosed to detect a potential PA following the appearance of nonspecific symptoms such as hematuria, hemospermia, lower urinary tract symptoms (LUTS), urinary retention and fever. This subtype is the most frequent because of its clinical presentation during the fifth or

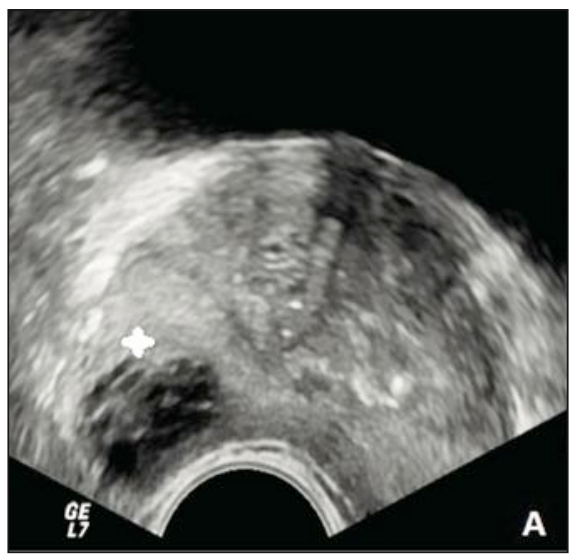

Figure $1 A$.

TRUS shows in transverse scan a prostatic abscess $(12 \mathrm{~mm})$ that interests one prostate lobe in the peripheral zone of the gland. It presents a discrete low echoic area with irregular contour (Type IIA).

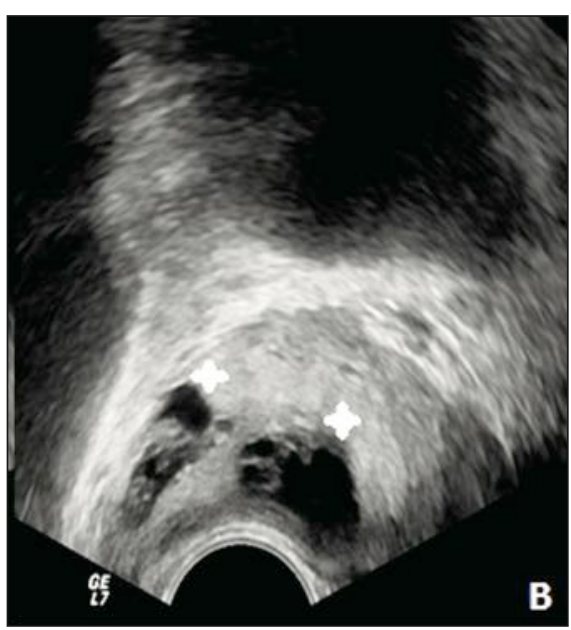

Figure 1B.

TRUS shows in longitudinal scan two prostatic abscesses $(>10 \mathrm{~mm})$ in the transitional and peripheral zone of the gland (Type IV). sixth decade of life and constitutes approximately 0.5$1 \%$ of patients hospitalized for prostatic disorders (10). Often Type I is considered to be a sequel of an acute o chronic prostatitis that has either not been treated or that has been treated inappropriately (11).

Type II - Prostatic abscess is present in a prostate lobe $(>10 \mathrm{~mm})$ and/or partially overcrosses the midline prostatic glandular border.

If the prostatic abscess interests one prostate lobe (> 10 $\mathrm{mm}$ ) focally or overcrosses the midline prostatic glandular border (Type II), it can be frequently located in the transition zone, as well as in the peripheral zone of the gland $(5,12)$. We can divide it into three subtypes (IIA, IIB and IIC) on the basis of US pattern and the type of clinical symptoms. The subtypes IIA and IIB have the same ultrasound pattern of subtypes IA and IB, although dimensions are $\geq 10 \mathrm{~mm}$ and/or extended in the contralateral part of the prostate gland (Figure 1A, B). These PA subtypes are diagnosed incidentally during a routine ultrasound. In the subtype IIC, PA can appear with the characteristics of both subtypes IIA and IIB, but it is diagnosed to detect a potential PA following the appearance of nonspecific symptoms such as hematuria, hemospermia, LUTS, urinary retention, dysuria, a palpably enlarged and tender prostate gland, fever, and sepsis. Subtypes IC and IIC are more often haemorrhages rather than infectious prostate biopsy's complication.

Hemorragic post-biopsy PA are rare with an incidence of $1.3 \%$ (12). It is not possible to differentiate by TRUS the sonographic distinctive features of the two conditions, although often the patient's medical history or a multiparametric MRI are helpful.

Type III - Prostatic abscess is present in both glandular lobes form of multifocal areas $(\leq 10 \mathrm{~mm})$.

If PA interests both glandular lobes by two or more multifocal areas each of size $\leq 10 \mathrm{~mm}$ (Type III), we can divide it into two subtypes (IIIA, IIIB) on the basis of ultrasound pattern and of clinical symptoms (Figure 2). In the subtype IIIA, PA presents a hypoechoic or anechoic or non-homogeneous content with irregular rim (possible thickened aspect or hyperechoic echostructure). This subtype of PA is diagnosed incidentally during a routine ultrasound. In Type IIIB, PA can appear with the US patterns of subtype IIIA, but this subtype is diagnosed to detect a potential PA following the appearance of symptoms (hematuria, hemospermia, LUTS, urinary retention) or in the case of painful and palpably tender prostate gland. This subtype is the most frequent cause of clinical presentation of granulomatous prostatitis. The exact aetiology of granulomatous prostatitis remains unclear and in many cases it may be idiopathic (13), although it can be also caused by several specific (as Mycobacterium tuberculosis) (14) and non-specific infectious agents. It can also be secondary to prostatic surgery such as transurethral resection (15). However, the areas of abnormally low hypoechoic or non-homogeneous structure occurring in granulomatous prostatitis have no characteristic ultrasonography pattern to differentiate them from cancer and histological confirmation is necessary to obtain a definitive diagnosis (16) 


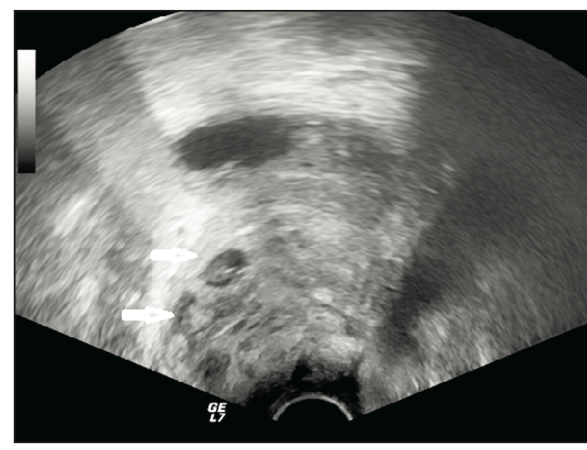

Figure 2.

TRUS shows in transverse scan multiple prostatic abscesses $(\leq 10 \mathrm{~mm})$ involving both lobes (Type III).).

Type IV - Prostatic abscess is present in both glandular lobes form of multifocal areas (>10 $\mathrm{mm}$ ).

If PA interests both glandular lobes by two or more multifocal areas each of size $>10 \mathrm{~mm}$ (Type IV). This type can be divided in the IV A and IV B according to the appearance of the clinical symptoms. The most common TRUS finding in all patients was the detection of a hypoechoic area with non-homogeneous structure. In some patients irregular contour and hyperechoic areas were additionally described (17). In literature and in our experience the PA Type IV is related to a massive caseous necrosis induced in patients that had received intravesical instillations of Bacillus Calmette-Guerin (BCG) for superficial bladder tumor (18-20).

Type V - Prostatic abscess involving intra or extraprostatic structures (bladder, urethra, seminal vesicles and prostatic capsule).

If the prostatic abscess, regardless of its size, presents compressive phenomena involving intra or extraprostatic structures as bladder, urethra, seminal vesicles and/or prostatic capsule (Type V), it always causes clinical symptoms due to extrinsic compression (6) of the structures listed as: hemospermia, hematuria, purulent urethral secretions, urinary retention, dysuria, incomplete bladder emptying or urinary urgency, and sometimes fever. Although fluctuation to the rectum should make one suspect abscess, this is not a constant finding, as in the case of para-urethral or bladder localization (Figure 3). Treatment of PA implied parental broad-spectrum antibiotic administration and/or abscess drainage. This may be performed, depending on its localization by transrectal or transperineal ultrasound, by perineal route, transurethral incision or resection of the prostate, or open perineal drainage (2).

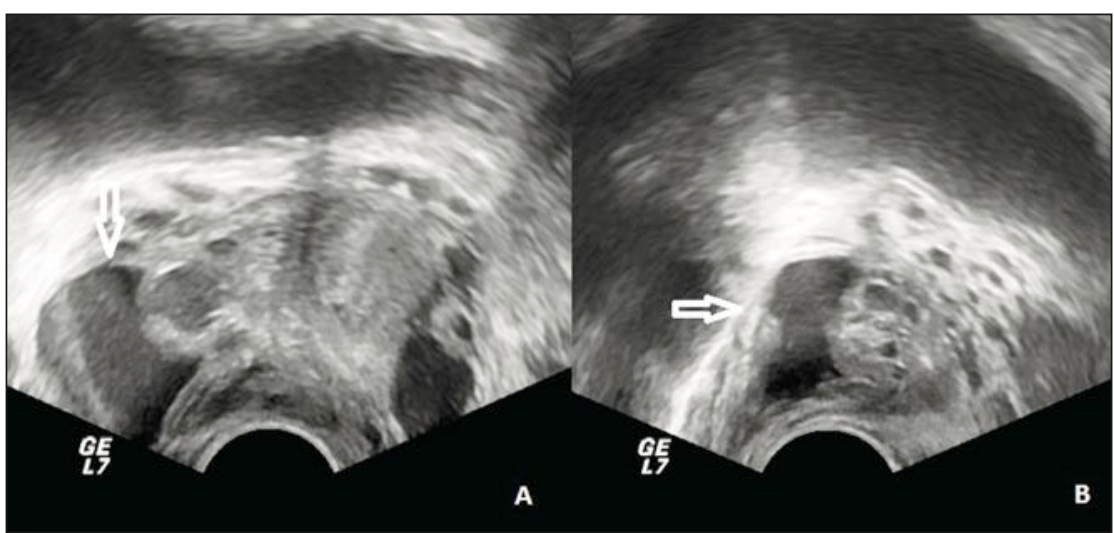

\section{Discussion}

PA is uncommon and difficult to diagnose because initial clinical presentation may mimic several other diseases of the lower urinary tract. Its incidence has markedly decreased for the widespread use of antibiotics (9). PA should be suspected in patients presenting with fever and persistent LUTS that do not respond to antibiotics. Predisposing factors for the development of PA are diabetes mellitus, bladder outlet obstruction, indwelling catheter, prostate biopsy, chronic renal failure, chronic liver disease and HIV infection $(2,21)$. The diagnosis and localization of PA have been facilitated with the advent of TRUS (22). Transabdominal ultrasonography is usually not sufficient to make a diagnosis because it does not delineate the anatomical details of PA, owing to its peripheral glandular location or to the physical constitution of the patient. TRUS can exactly define the localization, number and size of PA and help in the management and follow-up. However, according to some authors TRUS is not feasible in all patients as it is highly painful in presence of PA, and MRI or CT scan are more suitable to define the disease (23). In our experience the use of local anesthesia with lidocaine spray, allowed to perform TRUS in all patients in a comfortable way, except in a few conditions that might be a contraindication such as in patients with severe haemorrhoids or anal fistulas or submitted abdominoperineal resection (7). The most common finding of PA is a hypoechoic or anechoic lesion with more or less defined edges and a peripheral hyperechoic halo. A remarkable finding is the alteration of prostate ultrasound anatomy. However, a differential diagnosis must be made for a similar ultrasound appearance of tumors, cystic lesions, and focal acute prostatitis (8). Prostatic imaging with CT and MRI is important in the differential diagnosis of PA. Some studies shown that the more cost-effective CT and MRI hold no advantage over TRUS unless the abscess has penetrated the confines of the prostate (Type V) or there are further abscess foci suspected $(24,25)$. Therefore, a histological confirmation is necessary to obtain a definitive diagnosis in doubtful cases. TRUS is the most widely used technique in the guide for percutaneous aspiration or PA biopsy and in evaluation of response to treatment $(1,2,4,9)$.

In this study the classification of PA into five distinct types proposed is based on ultrasonography pattern and clinical evidence of the disease as reported in numerous studies. However, most published data regarding PA are case reports, and there is no standardization of the diagnostic and therapeutic routines. In review articles, the summary of several individual experiences permits to delineate some lines of action for PA.

Figure 3.

TRUS shows in transverse $(A)$ and longitudinal (B) scan prostatic abscesses (Type $V$ ) interesting more multifocal areas with inhomogeneous content and irregular rim bulging the capsule and urethra (white arrows). 
In the literature, few authors as Lim et al. (25) or Gorus et al. (26) tried to classify the pathology based on the ultrasound images acquired with TRUS but with the purpose to standardize a treatment of percutaneous aspiration rather than a clinical and ultrasonographic classification of the disease. Barozzi et al. (27) described the sonographic features of their patients related to bacterial etiology after abscess drainage of the gland, but not making a standardized imaging classification. Limitations of this classification could perhaps be identified in the lack of comparison with pathological features after biopsy. Additional studies with more detailed exposure measurement are warranted to evaluate questions about ultrasound imaging, pathology features, etiology and the best management for the treatment and therapy duration.

\section{Conclusions}

In last years prostatic imaging modalities (TRUS, CT and MRI) gained a wide acceptance in diagnosis of PA. Of these imaging modalities TRUS is widely used in diagnosis of PA for the low cost and low radiation exposure (28). The sonographic pattern of PA is usually characteristic and easily differentiated from other glandular lesions. The purpose of the study was to associate the use of TRUS to a clinical classification standardized to facilitate the diagnosis and localization of PA in order to direct the clinician to the correct management and adequate therapeutic treatment. Other studies will be required to verify its validity and clinical utility and to implement it.

\section{References}

1. Granados EA, Riley G, Salvador J, Vincente J. Prostatic abscess: diagnosis and treatment. J Urol. 1992; 148:80-82.

2. Oliveira P, Andrade JA, Porto HC, Filho JE, Vinhaes AF. Diagnosis and treatment of prostatic abscess. Int Braz J Urol. 2003; 29:30-34.

3. Langer JE, Cornud F. Inflammatory disorders of the prostate and the distal genital tract. Radiol Clin North Am. 2006; 44:665-677.

4. Varkarakis J, Sebe P, Pinggera GM, Bartsch G, Strasser H. Threedimensional ultrasound guidance for percutaneous drainage of prostatic abscesses. Urology. 2004; 63:1017-1020.

5. Afaq A, Koh DM, Padhani A, et al. Clinical utility of diffusionweighted magnetic resonance imaging in prostate cancer. BJU Int. 2011; 108:1716-1722

6. Bayne CE, Davis WA, Rothstein CP, Engel JD. Seminal vesicle abscess following prostate biopsy requiring transgluteal percutaneous drainage. Can J Urol. 2013; 20:6811-6814.

7. Dell'Atti L. Lidocaine spray administration transrectal ultrasound guided prostate biopsy: five years of experience. Arch Ital Urol. 2014; 86:340-343.

8. Galosi AB, Montironi R, Fabiani A, et al. Cystic lesions of the prostate gland: an ultrasound classification with pathological correlation. J Urol. 2009; 181:647-657.

9. Jang K, Lee DH, Lee SH, Chung BH. Treatment of prostatic abscess: case collection and comparison of treatment methods. Korean J Urol. 2012; 53:860-864.

10. Collins SM, Correa H, Ortenberg J. Prostatic abscess in the newborn: an unrecognized source of urosepsis. Urology. 2001; 57:554.
11. Collado A, Palou J, García-Penit J, et al. Ultrasound-guided needle aspiration in prostatic abscess. Urology. 1999; 53:548-552.

12. Herranz Amo F, Verdú Tartajo F, Díez Cordero JM, et al. Hemorrhagic prostatic cyst following ultrasound guided biopsy. A case report. Arch Esp Urol. 1999; 52:379-80.

13. Naik KS, Carey BM. The transrectal ultrasound and MRI appearances of granulomatous prostatitis and its differentiation from carcinoma. Clin Radiol. 1999; 54:173-175.

14. Gorse GJ, Belshe RB. Male genital tuberculosis: a review of the literature with instructive case reports. Rev Infect Dis. 1985; 7:511-524.

15. Carrero López VM, Rodríguez Antolín A, Caballero J, et al. Granulomatous prostatitis. An infrequent diagnosis. Review of our series. Actas Urol Esp. 1994; 18:77-84.

16. Liu S, Miller PD, Holmes SA, et al. Eosinophilic prostatitis and prostatic specific antigen. Br J Urol. 1992; 69:61-63.

17. Bour L, Schull A, Delongchamps NB, et al. Multiparametric MRI features of granulomatous prostatitis and tubercular prostate abscess. Diagn Interv Imaging. 2013; 94:84-90.

18. Aust TR, Massey JA. Tubercular prostatic abscess as a complication of intravesical bacillus Calmette-Guérin immunotherapy. Int J Urol. 2005; 12:920-921.

19. Caulier P, Yombi JC, Dufaux M, et al. Prostate abscess following intravesical BCG therapy. Acta Clin Belg. 2009; 64:436-437.

20. Matlaga BR, Veys JA, Thacker CC, Assimos DG. Prostate abscess following intravesical bacillus Calmette-Guerin treatment. J Urol. 2002; 167:251.

21. Trauzzi SJ, Kay CJ, Kaufman DG, Lowe FC. Management of prostatic abscess in patients with human immunodeficiency syndrome. Urology. 1994; 43:629-633.

22. Cytron S, Weinberger M, Pitlik SD, Servadio C. Value of transrectal ultrasonography for diagnosis and treatment of prostatic abscess. Urology 1988; 32:454-458

23. Ludwig M, Schroeder-Printzen I, Schiefer HG, Weidner W. Diagnosis and therapeutic management of 18 patients with prostatic abscess. Urology 1999; 53:340-345.

24. Somuncu I, Saglam M, Yagci S, et al. Multiloculated prostate abscess: treatment with transrectal ultrasound guided transrectal needle aspiration and lavage with the saline and antibiotic. Clin Imaging. 2003; 27:251-255.

25. Lim JW, Ko YT, Lee DH, et al. Treatment of prostatic abscess: value of transrectal ultrasonographically guided needle aspiration. J Ultrasound Med. 2000; 19:609-617.

26. Gögüs C, Ozden E, Karaboga R, Yagci C. The value of transrectal ultrasound guided needle aspiration in treatment of prostatic abscess. Eur J Radiol. 2004; 52:94-98.

27. Barozzi L, Pavlica P, Menchi I, De Matteis M, Canepari M. Prostatic abscess: diagnosis and treatment. AJR Am J Roentgenol. 1998; 170:753-757.

28. Dell'Atti L. Prostatic abscess after transrectal ultrasound-guided prostate biopsy. Case report. G Chir. 2013; 34:260-262.

\section{Correspondence}

Lucio Dell'Atti, MD, PhD (Corresponding Author) dellatti@hotmail.com

Department of Urology, University Hospital "St.Anna".

8 A. Moro Street, 44124 Cona - Ferrara, Italy 